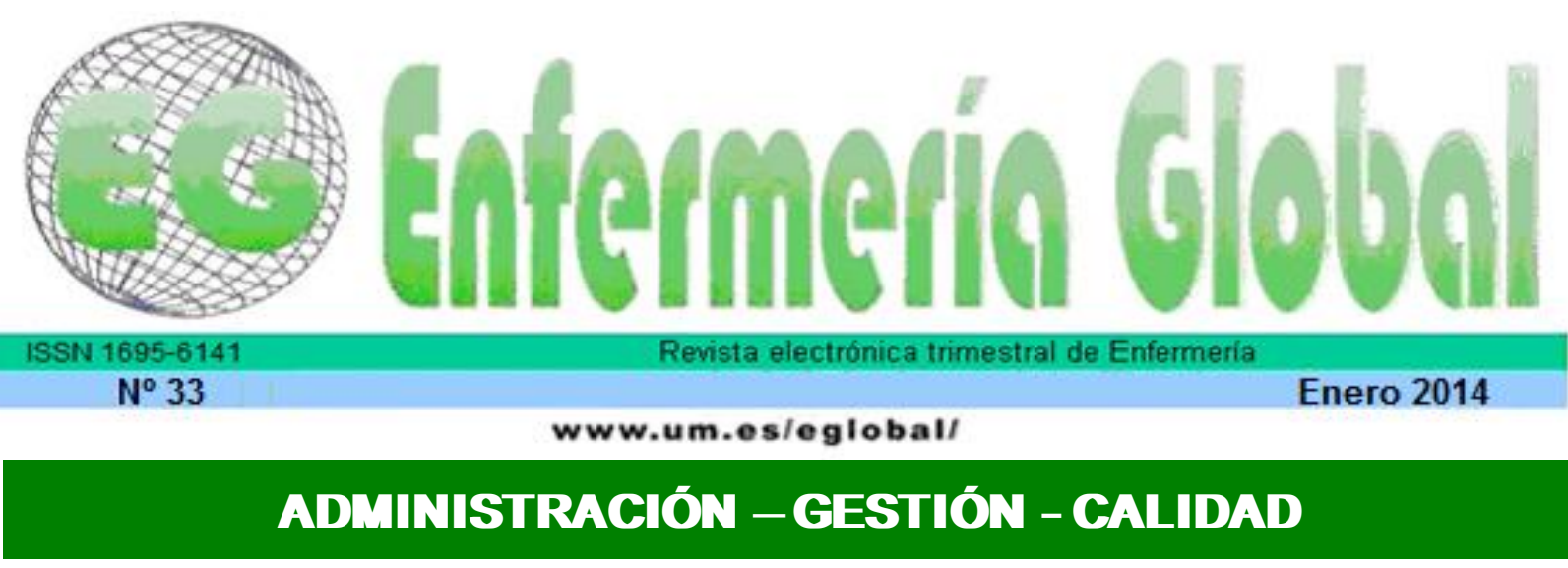

\title{
Síndrome de Burnout en el personal de enfermería en UVI
}

Burnout syndrome in nursing staff in ICU

\author{
*Sánchez Alonso, Patricia **Sierra Ortega, Víctor Manuel \\ *Enfermera/o, UVI médica Hospital Ramón y Cajal, Madrid. E-mail: patry_ss@hotmail.com
}

Palabras clave: Burnout; UVI; enfermería; MBI; paciente crítico

Keywords: Burnout; ICU; nursing; MBI; critical patient.

\section{RESUMEN}

Introducción: El síndrome de Burnout aparece cuando se mantiene una relación de ayuda con una relación interpersonal intensa con los beneficiarios del trabajo. En numerosos estudios se ha considerado al personal de enfermería como grupo vulnerable. Algunos estudios revelan que las unidades que generan mayor estrés son aquellas que reúnen pacientes críticos o terminales. El ambiente laboral de estas unidades, las continuas demandas físicas y emocionales a las que las enfermeras se ven sometidas por parte de los pacientes y la ambigüedad en su rol son características que predisponen al desarrollo de dicho síndrome.

Objetivos: El objetivo principal es determinar el grado de Burnout en el equipo de enfermería de la UVI médica del hospital Ramón y Cajal.

Material y métodos: Muestra de 68 enfermeros y auxiliares que trabajan en la UVI. Como instrumento, se utiliza el cuestionario Maslach Burnout Inventory en su versión en español, de manera autoadministrada.

Resultados: Bajas puntuaciones en las tres dimensiones del síndrome (cansancio emocional, despersonalización y realización personal), y la mayoría de la población, un 45,6\%, tendencia a padecerlo.

Conclusiones: El perfil del trabajador que padece síndrome de Burnout sería: enfermera, de 35 a 44 años, con contrato fijo, experiencia laboral mayor de 16 años y una antigüedad de 1 a 5 años en el puesto de trabajo.

\section{ABSTRACT}

Introduction: The burnout syndrome occurs when a supportive relationship is maintained with a strong interpersonal relationship with the beneficiaries of that work. Several studies have considered the nursing staff as a fragile group. Some studies revealed that units that generate greater stress are those that meet critical or terminal patients: the environment, physical and emotional continuing demands to the nurses who are subjected by patients, and role ambiguity are characteristics that predispose the development of this syndrome. 
Objectives: The main objective is to determine the degree of burnout in the team ICU nursing Hospital Medical Ramón y Cajal.

Material and methods: Sample of 68 nurses and assistants working in the ICU. As an instrument, we used Maslach Burnout Inventory in its Spanish version, self administered.

Results: Low scores on the three dimensions of the syndrome (emotional exhaustion, depersonalization and personal accomplishment), and most sample with a $45.6 \%$, tendency to suffer.

Conclusions: Profile of workers suffering burnout is: nurse, 35 to 44 years, with contract fixed, experience over 16 years and an age of $1-5$ years in that job.

\section{INTRODUCCIÓN}

Los profesionales sanitarios (enfermeros y auxiliares de enfermería en nuestro caso), constituyen un colectivo que se haya en continuo contacto con personas que tienen problemas o motivos de sufrimiento ${ }^{(1)}$. A veces se implican tanto en su trabajo, que pueden llegar a agotarse física y psíquicamente, de tal forma que resultan ineficaces en el desempeño adecuado de sus actividades laborales ${ }^{(2)}$.

El término Burnout se refiere a una situación de agotamiento emocional, cada vez más frecuente entre los profesionales que prestan sus servicios a través de una relación directa y sostenida con las personas, que son las beneficiarias del trabajo, entre las que están todos los profesionales sanitarios ${ }^{(3,4)}$.

La definición del burnout más consolidada es la de Maslach y Jackson en $1981^{(5)}$, quienes consideran que es una respuesta inadecuada a un estrés emocional crónico cuyos rasgos principales son: agotamiento físico y/o psicológico, una actitud fría y despersonalizada en la relación hacia los demás y un sentimiento de inadecuación a las tareas que ha de realizar ${ }^{(4,6,7,8)}$.

El burnout y el estrés laboral conllevan el deterioro de las condiciones de trabajo, el aumento de la accidentalidad y el aumento del absentismo ${ }^{(2)}$.

Las circunstancias mencionadas se dan, comúnmente, en las áreas de cuidado crítico de un hospital donde hay pacientes en situaciones límites, de estado de emergencia continua e inestabilidad permanente, que demandan una intervención rápida y efectiva por parte del profesional de la salud, quienes deben trabajar en un ambiente impersonal y opresivo, extremada y altamente tecnificado, con ruidos múltiples generados por alarmas, quejidos y problemas de espacio, tiempo y consecuente sobrecarga laboral. Todo ello crea un clima psicológico de ansiedad, no sólo en el equipo asistencial, sino también en los pacientes y familiares, especialmente en personal de enfermería, que está en la primera línea de asistencia ${ }^{(3)}$, incrementando el riesgo de padecer burnout ${ }^{(7)}$.

En este sentido, la enfermería se ha identificado como un grupo especial de riesgo porque en ella concurren factores personales, profesionales e institucionales, que son causas potenciales del síndrome que estudiamos ${ }^{(5,7)}$.

En este marco, los profesionales de enfermería son extremadamente vulnerables a desarrollar síndrome de Burnout. Estudios citados por Albadejo et al. ${ }^{(7)}$ señalan que aproximadamente el $25 \%$ de las enfermeras estarían afectadas por el síndrome. Además, las condiciones laborales y las características del tipo de trabajo que 
desarrollan los enfermeros en las UCls, hacen de este un grupo de riesgo para el desarrollo de dicho síndrome ${ }^{(4)}$.

El cuestionario ha sido el método mas empleado tradicionalmente para estimar el síndrome y en concreto el MBI de Maslach y Jackson (1981). El MBI plantea al sujeto una serie de enunciados sobre los sentimientos o pensamientos con relación a su interacción con el trabajo ${ }^{(7,9)}$.

Dicho cuestionario consta de tres subescalas: cansancio emocional, despersonalización y realización personal ${ }^{(10)}$.

En numerosos trabajos se ha considerado al personal de enfermería como grupo vulnerable. Algunos estudios revelan que las unidades que generan mayor estrés son aquellas que reúnen pacientes críticos o terminales. El ambiente laboral de estas unidades, las contínuas demandas físicas y emocionales a las que las que las enfermeras se ven sometidas por parte de los pacientes y la ambigüedad en su rol son características que predisponen al desarrollo de dicho síndrome ${ }^{(4)}$. A estos factores hay que añadir que cada vez es mayor el tiempo que los enfermeros pasan cuidando de los pacientes, debido al déficit económico que presenta el sistema sanitario, con la consecuente carga de trabajo (expresiones verbales de los encuestados).

El objetivo principal de nuestro estudio es estimar la prevalencia del síndrome de Burnout y cada una de sus dimensiones en el personal de enfermería en la unidad de cuidados intensivos (UVI médica) del Hospital Ramón y Cajal, en la provincia de Madrid.

\section{MATERIAL Y MÉTODO}

Estudio observacional descriptivo, transversal, con muestreo aleatorio simple. La muestra de estudio está compuesta por todo el equipo de enfermería que trabaja en la UVI médica del Hospital Ramón y Cajal, de las cuales 68 rellenan el cuestionario de manera correcta $(\mathrm{N}=68)$, (Ver anexo l: Características generales de la muestra). Los criterios de inclusión son: Enfermeros y auxiliares de enfermería que trabajan en la UVI médica del Hospital Ramón y Cajal en el turno de mañana, tarde o noche durante el periodo de recogida de datos, y que rellenen el cuestionario de manera correcta.

Uno de los instrumentos utilizados para la recogida de datos ha sido un cuestionario donde se recogen datos sociodemográficos de la muestra de estudio. Consta de ocho preguntas cerradas y opción múltiple: categoría, sexo, edad, estado civil, tipo de contrato, experiencia laboral, antigüedad en el puesto de trabajo y el tipo de turno.

El segundo instrumento de recogida de datos ha sido el cuestionario Maslach Burnout Inventory $(\mathrm{MBI})$ en su versión validada en español.El cuestionario consta de 22 preguntas valoradas en una escala tipo likert con cuatro posibles respuestas ${ }^{(9)}$, y mide tres dimensiones del síndrome de Burnout ${ }^{(8,11,12)}$ :

- Cansancio emocional: preguntas 1, 2, 3, 6, 8, 13, 14, 16, 20. Valora la vivencia de estar exhausto emocionalmente por las demandas del trabajo. Puntuación máxima 54 puntos. 
- Despersonalización: preguntas 5, 10,11, 15, 22. Valora el grado en que cada uno reconoce actitudes de frialdad y distanciamiento. Puntuación máxima 30 puntos.

- Realización personal: preguntas 4, 7, 9, 12, 17, 18, 19, 21. Evalúa los resultados de autosuficiencia y realización personal en el trabajo. Puntuación máxima 48.

Para identificar el síndrome de burnout se realiza la suma de las tres dimensiones de la encuesta ${ }^{(13)}$ y sería preciso obtener puntuaciones altas en cansancio emocional y despersonalización y bajas en realización personal ${ }^{(3)}$ (Ver anexo II).

El material se distribuyó entre el personal del Servicio de la UVI médica durante el mes de agosto y septiembre de 2012, tanto en turno de mañana, de tarde y de noche. Se administraron y recogieron las encuestas personalmente. No se administró ningún consentimiento informado, ya que la participación en el estudio es voluntaria, y al rellenar el cuestionario, los participantes ofrecen su consentimiento.

\section{RESULTADOS}

\section{Cansancio emocional (Anexo III)}

El $19,1 \%$ de la muestra presenta un nivel alto de CE, el 20,6\% un nivel medio y el $60,3 \%$ un nivel bajo.

El $47 \%$ de los enfermeros y el $13 \%$ de los auxiliares presentan bajo CE, siendo la mayoría mujeres (52,9\%), mientras que el $13,2 \%$ de los enfermeros y el $5,8 \%$ de los auxiliares presentan niveles altos.

El rango de edad donde mayor cansancio emocional se experimenta es la comprendida entre 35 y 54 años con un 14,6\%.

El $30,8 \%$ de los eventuales presentan puntuaciones bajas, frente al $26,6 \%$ de los fijos y el $2,9 \%$ de los interinos. El $10,3 \%$ de los fijos experimental alto CE.

Se observa aumento de CE con forme avanza la experiencia laboral, dándose el mayor porcentaje de alto CE en trabajadores con experiencia mayor de 21 años, representando el $7,3 \%$ de la muestra. El $35,7 \%$ de las puntuaciones altas de CE (representadas por el $7,3 \%$ de la muestra total) corresponde a trabajadores con antigüedad en UVI entre 1 y 5 años.

\section{Despersonalización (Anexo IV)}

El $23,5 \%$ de la muestra presenta un nivel de DP alto, un $30,9 \%$ un nivel medio y el $45,6 \%$ un nivel bajo.

El $33,8 \%$ de los enfermeros y el $11,7 \%$ de los auxiliares de enfermería presentan puntaciones bajas. Las puntuaciones altas están representadas por el 16,1\% para los enfermeros y $7,4 \%$ para auxiliares de enfermería.

El $47 \%$ de las mujeres presentan puntuaciones altas y medias mientras que ningún hombre ha obtenido puntuaciones altas y un $7,3 \%$ puntuaciones medias. 
Puntuaciones altas para la dimensión de DP son más frecuentes en los rangos de edad de 25-34 y 35-44 años. El grupo de edad de 55 años y más se ve menos afectado al no presentar ningún participante con puntuación alta. Las puntuaciones bajas de despersonalización superan a las puntuaciones altas en un $22 \%$.

El resultado que más se repite son puntuaciones bajas, que han sido obtenidas por personal fijo en un 20,5\%, seguido de personal eventual con un $19,2 \%$ de la muestra. En cuanto a la experiencia laboral, presentan puntuaciones similares todos los grupos. Los grupos con menos número de puntuaciones altas, son el grupo de 11-15 años, que representa $1,6 \%$ y el grupo con $16-20$ años que representa el $4,5 \%$. El resto de los grupos representan cada uno el 5,8\%. El mayor número de puntuaciones bajas se repiten en el grupo de 16-20 años, representando el 14,8\%.

El personal que lleva entre 1 y 5 años trabajando en la UVI es el que presenta mayor número de puntuaciones altas en DP, representado por el $10,2 \%$ de la muestra, y la mitad de los participantes de este rango. Las personas con antigüedad menor de 1 año y con 21 o más años trabajados en este servicio, presentan la misma cantidad de resultado de DP altos, representado por el 5,8\% de la muestra para cada grupo. Los grupos que han presentado mayor número de puntuaciones bajas para DP han sido los que han trabajado menos de 1 año en el servicio, con el $16 \%$ de la muestra; y los que han trabajado entre 11 y 15 años con un $11,7 \%$.

\section{Realización personal (Anexo V)}

El 39,9\% presenta puntuaciones bajas, el 30,9\% puntuaciones medias y el $29,4 \%$ puntuaciones altas.

El $29,4 \%$ y $26,4 \%$ de los enfermeros presentan puntuaciones bajas y medias respectivamente. Sin embargo, los auxiliares de enfermería presentan en la misma proporción puntuaciones altas y bajas, con 10,3\% de la muestra para cada resultado.

La mayor parte de las mujeres obtienen puntuaciones bajas representado por el $35,4 \%$ de la muestra; y los hombres puntuaciones medias en un $7,3 \%$.

El rango de edad con menos RP es de 35 y 44 años y de 45 y 54 años, obteniendo puntuaciones bajas para esta dimensión el $10,2 \%$ y el $14,7 \%$ de la población de estudio. También se dan puntuaciones altas con mayor frecuencia en el grupo de edad de 35-44 años. Bajas puntuaciones de RP superan a altas puntuaciones en un $10,2 \%$.

Del total de la muestra con puntuaciones bajas en esta dimensión, un 20,5\% corresponde a personal fijo, y un $14,7 \%$ a personal eventual. Los interinos representan el $4,4 \%$ en las puntuaciones bajas para esta dimensión. También es el personal fijo quien representa el mayor número de altas puntuaciones en RP.

Los grupos con experiencia menor de 5 años y con 21 años y más son los grupos que presentan mayor número de bajas puntuaciones de $\mathrm{RP}$, representados por un $10,3 \%$ de la muestra para cada uno. El grupo con experiencia comprendida entre 16 y 20 años es el que ha obtenido mayor número de puntuaciones altas en $\mathrm{RP}$, representado por un $11,7 \%$ de la muestra.

El mayor número de puntuaciones bajas para la realización personal se da en los grupos con menos de 1 año trabajado en la UVI, con antigüedad entre 1 y 5 años y 
con 21 años y más, estando representado en cada grupo por el 10,3\% de la muestra; y siendo en este último grupo además el que más puntuaciones medias ha obtenido, con un $8,8 \%$. El personal con mayor número puntuaciones altas para RP corresponde a los que tienen antigüedad en el servicio de 11 a 15 años, representado por el 10,3\% de la muestra.

En nuestra muestra de estudio, esta dimensión es la más afectada.

\section{Fase de sindrome de Burnout (Anexo VI)}

El grado de ausencia, tendencia y síndrome de Burnout se distribuye entre la muestra de estudio según indica el gráfico 1.

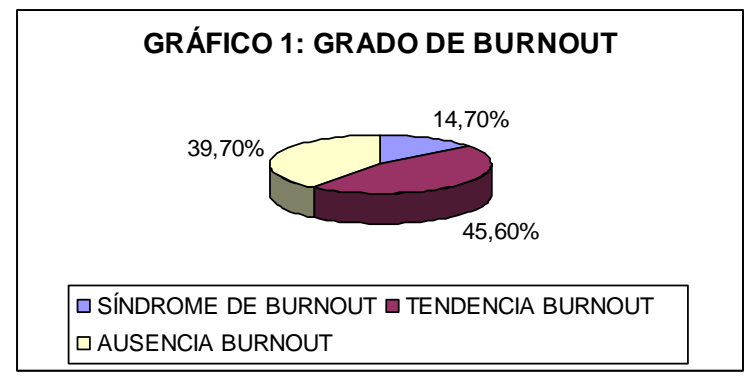

El 11,7\% de la muestra está representada por enfermeros con síndrome de Burnout, observándose además un gran número personas con tendencia a padecerlo, en concreto un $45 \%$ de las enfermeras, que representan un $34 \%$ de la muestra total. Los auxiliares con SB representan el 3\% de la muestra, estando muy igualados los resultados de $\mathrm{TB}$ y $\mathrm{AB}$ con un 11,7\% y un 10,3\% respectivamente. Mientras que las mujeres con SB representan el $14,7 \%$ de la muestra, y un $36,7 \%$ (42\% de las mujeres) presentan TB, no hay ningún hombre de la muestra que lo padezca, a pesar de ello el $66,6 \%$ de los hombres que representan el $8,8 \%$ de la muestra presentan tendencia; y tan sólo el 33,4\% representado por el 4,4\% de la muestra total presenta ausencia de burnout. No hay diferencias significativas entre sexos $(p=0,2668)$.

El $14,7 \%$ de la muestra presenta síndrome de Burnout, de los cuales el $3 \%$ lo padecen personas entre 25 y 34 años, el 7,3\% personas entre 35 y 44 años y el $4,4 \%$ personas entre 45 y 54 años. La tendencia a burnout representa el $45,6 \%$ de la muestra, de los cuales $10,3 \%$ lo padecen personal entre 25 y 34 y, 35 y 44 años, y el $16,1 \%$ personal entre 45 y 54 años. El grupo con mayor número de ausencia de burnout corresponde a personas entre 35 y 44 años, representando un 13,2\% del total de la muestra.

El personal fijo presenta mayor número de personas con SB representando el 17,1\% del total de personal fijo y suponiendo el $8,8 \%$ de la muestra de estudio. También es el grupo donde se da mayor número de personas con TB, representando el $26,5 \%$ del total de la muestra, y superando a las personas con TB en el personal eventual en un $8,8 \%$. A pesar de presentar un gran número de personas con TB, el personal eventual es el grupo donde predominan los trabajadores con $A B$, representado por el $19,1 \%$ de la muestra, y suponiendo un $3 \%$ mayor que el grupo de personas fijas, siendo este además más numeroso que el primero.

El SB es más frecuente en las personas con más de 15 años de experiencia, hallándose en este grupo un 4,4\% de la muestra para el rango de 16-20 años y para 
21 años o más de experiencia. También es el grupo con una experiencia de 21 años y más el que mayor número de personas con TB, representando un $8,9 \%$ más que en personas con experiencia inferior a 5 años, rango de edad con mayor número de personas con TB, después del rango anterior. El mayor número de encuestas con resultado de ausencia de burnout se encuentra en las personas con una experiencia laboral menor de 5 años, y en los que han trabajado entre 16 y 20 años. No hay diferencias significativas entre los grupos $(p=0,1499)$.

El mayor número de personas con SB tienen una antigüedad en la UVI médica entre 1 y 5 años y las personas con antigüedad en el puesto de 21 años y más. Las personas con TB están repartidas de forma equitativa entre los grupos, suponiendo el grupo de 21 años y más el que mayor número de personas con TB presenta, representando el $13,2 \%$ de la muestra, y suponiendo el $60 \%$ de las personas que pertenecen a ese grupo. El grupo con antigüedad en el puesto menor de 1 año es el que presenta mayor número de personas con $A B$, suponiendo un $16,1 \%$. No hay diferencias significativas $(p=0,4080)$.

\section{DISCUSIÓN}

En primer lugar, hacemos referencia a la alta participación que hemos tenido por parte de la muestra de estudio, al igual que en el estudio de Puialto Durán et al $l^{(12)}$, Solano Ruíz et al. ${ }^{(4)}$ con una tasa de respuesta de un $72,31 \%$ y un $77,6 \%$ respectivamente, no llegando a alcanzar el 100\% de participación, como en nuestro caso. Por otro lado, están los estudios de Díaz Muñoz ${ }^{(8)}$, Meseguer et al. ${ }^{(14)}$, y Gil-Monte et al. ${ }^{(15)}$, en los que el nivel de participación es bajo. Hemos de mencionar que los tres últimos estudios han sido realizados en 1991 y 1996. De este dato podemos concluir que los estudios más recientes presentan mayor participación, y menos reticencia a la hora de contestar.

Coincidimos con el estudio de Puialto Durán et al. ${ }^{(12)}$ en que una parte de la muestra hicieron referencia a la dificultad para cuantificar la frecuencia con que presentan los ítems del cuestionario MBI.

Otro punto coincidente con la bibliografía consultada es el sexo de la muestra, representado en el estudio de Puialto Durán et al. ${ }^{(12)}$ por el $98 \%$, en el estudio de Solano Ruiz et al. ${ }^{(4)}$ por el $80,7 \%$, en el de Albadejo et al. ${ }^{(7)}$ por el $89,1 \%$, estando nuestra muestra formada por $86,7 \%$ de mujeres.

Hay disparidad en cuanto a los resultados de síndrome de burnout y sus tres dimensiones.

Según el estudio de Frade et al. (1), las enfermeras y las auxiliares obtienen puntuaciones altas en síndrome de Burnout: $26 \%$ de la muestra. En el estudio de Albadejo et al. ${ }^{(7)}$ se obtienen puntuaciones de síndrome de burnout en un $30,5 \%$ de la muestra. En contra de nuestro estudio que representa un porcentaje más bajo, un $11,7 \%$, al igual que en el estudio de Rodríguez López et al. ${ }^{(11)}$, en el que síndrome de Burnout representa el $10,34 \%$ de la muestra, y el estudio de Ríos ${ }^{(16)}$ en un $12 \%$.

La mayoría de la población de estudio presenta tendencia de burnout en un porcentaje muy igualado con el estudio de Albadejo et al. ${ }^{(7)}$, que representa un $48,9 \%$. 
Según el estudio de Puialto Durán et al. ${ }^{(12)}$, la franja de edad con mayor frecuencia era entre 45-54 años, pero en nuestro estudio predomina la edad de 35 a 44 años, coincidiendo en la larga experiencia laboral, de más de 15 años y antigüedad en el puesto de más de 21 años. En el estudio de Solano Ruiz et al. ${ }^{(4)}$, se obtienen las mayores puntuaciones del MBI en los mayores de 44 años. Albadejo et al. ${ }^{(7)}$ afirma que el perfil de la persona con síndrome de burnout con una experiencia en el puesto mayor de 15 años. Sin embargo, Rodríguez López ${ }^{(11)}$ refiere que el personal con más antigüedad es inmune al los efectos del burnout. En nuestro estudio se observa un incremento del síndrome de burnout y tendencia a padecerlo con el paso del tiempo, pero se observa una disminución brusca en los mayores de 55 años.

Los estudios de Puialto Durán et al.(12), García Grau et al ${ }^{(17)}$, Ríos et al. ${ }^{(16)}$ y Frade et al. ${ }^{(1)}$ obtienen niveles altos para cansancio emocional, y niveles medios el estudio de Carmona et al. ${ }^{(18)}$, Díaz Muñoz ${ }^{(8)}$. En el otro extremo, la mayor parte de la muestra de nuestro estudio ha obtenido puntuaciones bajas, coincidiendo con el estudio de Albadejo el al. ${ }^{(7)}$, Gamonal et al. ${ }^{(3)}$, Solano Ruiz et al. ${ }^{(4)}$.

Coincidiendo Solano Ruiz et al. ${ }^{(4)}$, las mujeres presentan mayores puntuaciones en cansancio emocional, coincidiendo con Maslach et al. ${ }^{(5)}$, quien afirma que las mujeres obtienen mayores puntuaciones en esta dimensión asociadas a la socialización de roles.

Convenimos con Solano Ruiz et al. (4), en que los fijos presentan niveles más elevados de cansancio emocional, y con Albadejo et al. (7) que obtiene mejores resultado de en el síndrome de Burnout y sus dimensiones en el personal eventual, resultado opuesto al estudio de Carmona et al. ${ }^{(18)}$, quien refiere que las enfermeras contratadas presentan niveles más altos de cansancio emocional debido a la incertidumbre e inseguridad laboral.

En la dimensión de despersonalización, García Grau et al. ${ }^{(17)}$ y Santana et al. ${ }^{(19)}$ obtuvieron niveles altos. Díaz Muñoz ${ }^{(8)}$, Ríos et al. ${ }^{(16)}$, Puialto Durán et al. ${ }^{(12)}$, Albadejo et al. (7) presentan puntuaciones medias. Nuestro estudio presenta puntuaciones bajas, igual que el estudio de Solano Ruiz et al. ${ }^{(4)}$ y Gamonal et al. ${ }^{(3)}$.

En lo referente a la realización personal, el mayor porcentaje de nuestro estudio corresponde con puntuaciones bajas, coincidiendo con el estudio de García Grau et al. ${ }^{(17)}$, Arauco et al. ${ }^{(13)}$, Santana et al. ${ }^{(19)}$, Ríos et al. ${ }^{(16)}$, Albadejo et al. ${ }^{(7)}$, Gamonal et al. ${ }^{(3)}$. El estudio de Piualto Durán et al. ${ }^{(12)}$ presenta puntuaciones medias y el estudio de Solano Ruiz et al. ${ }^{(4)}$ puntuaciones altas. Por tanto, es esta dimensión la que más se ve afectada en la mayoría de la bibliografía consultada, como refiere Díaz Muñoz ${ }^{(8)}$, obteniéndose los resultados más llamativos.

Hacer referencia al estudio de Arauco et al. ${ }^{(13)}$, y al de Gamonal et al. ${ }^{(3)}$, pues son dos estudios de diferente contexto al nuestro, realizados en Perú, sin embargo, se observan resultados similares al del resto de bibliografía consultada, y a nuestro estudio.

Al igual que en el estudio de Solano Ruiz et al. ${ }^{(4)}$, no se obtienen diferencias significativas en el burnout. 


\section{CONCLUSIONES}

Como conclusión final afirmamos que los trabajadores de la UVI médica presentan bajas y medias puntuaciones en las tres dimensiones y que la mayoría de la población, 45,6\%, presenta tendencia a burnout, estando el síndrome de Burnout presente en bajo porcentaje. Esta afirmación se corresponde con la bibliografía estudiada, remarcando al personal que trabaja en este servicio como personal de riesgo. La dimensión más afectada en nuestro estudio es la baja realización personal, seguido de una alta despersonalización, y por último un alto cansancio emocional.

Según los resultados obtenidos, el perfil del trabajador que padece síndrome de Burnout sería: enfermera, de 35 a 44 años, con contrato fijo, experiencia laboral mayor de 16 años y una antigüedad de 1 a 5 años en el puesto de trabajo.

\section{BIBLIOGRAFÍA}

(1) Frade Mera MJ, Vinagre Gaspar R, Zaragoza García I, Viñas Sánchez S, Antúnez Melero E, Álvarez González $S$ et al. Síndrome de burnout en distintas unidades de cuidados intensivos. Enferm Intensiva.2009; 20(4):131-40.

(2) Torres Morera LM. Tratado de cuidados críticos y emergencias. Tomo I. Madrid: Arán; 2001.

(3) Gamonal Mosaurieta YL, García Vargas CM, Silva Matheus Zl. Síndrome de burnout en el profesional de enfermería que labora en áreas críticas. Rev. enferm. Herediana. 2008;01(1): 33-39.

(4) Solano Ruiz MC, Hernández Vidal P, Vizcaya Moreno MF, Reig Ferrer A. Síndrome de Burnout en profesionales de enfermería de cuidados críticos. Enferm Intensiva. 2002; 13(1): 9-16.

(5) Maslach C, Jackson SE. The measurement of experienced burnout. Journal of Occupational Behaviour 1981; 2: 99-113.

(6) Martínez Pérez A. El síndrome de burnout. Evolución conceptual y estado actual de la cuestión. Vivat Academia. 2010; 112:1-40.

(7) Albadejo R, Villanueva R, Ortega P, Astasio P, Calle ME, Domínguez V. Síndrome de burnout en el personal de enfermería de un hospital de Madrid. Rev. Esp Salud Pública. 2004; 78(4):505-16.

(8) Díaz Muñoz-Crespo MJ. Síndrome del quemado en profesionales de Enfermería que trabajan en un hospital monográfico para pacientes cardíacos. Nure investigación [Internet] 2005 [Consultado: 27/07/2012]; 18: [7 páginas]. Disponible en:

http://www.fuden.es/FICHEROS ADMINISTRADOR/ORIGINAL/Original18.pdf.

(9) Llaneza Álvarez JF. Ergonomía y psicología aplicada. Manual para la formación del especialista. 12 ed. Valladolid: Lex Nova; 2009.

(10) Graciano E, Ferraz Bianca ER. Impacto del estrés ocupacional y burnout en enfermeros. Enf Global. 2010; 9(1): 1-20.

(11) Rodríguez López M, Fernández Barral R, Benítez Canosa MC, Castiñeira MJ, Brea Fernández AJ. Correlación entre carga de trabajo, síndrome de Burnout y calidad de vida en una unidad de críticos. Enf Global. 2008; 7(3): 1-11.

(12) Puialto Durán MJ, Antolín Rodríguez R, Moure Fernández L. Prevalencia del síndrome del quemad@ y estudio de factores relacionados en l@s enfermer@as del CHUVI (Complejo Hospitalario Universitario de Vigo). Enf Global. 2006; 5(1):1-18.

(13) Arauco OG, Enciso VJ, García MR. Factores sociodemográficos y síndrome de burnout en el profesional de enfermería del Hospital Nacional Arzobispo Loayza. Tesis de licenciatura. Lima, Perú: Universidad Peruana Cayetano Heredia, 2004. 
(14) Meseguer A, González R, Calatayud E. Enfermería Burnout y unidades de alto riesgo. Enferm Clínica. 1996; 6:111-24.

(15) Gil-Monte PR, Peiró JM, Valcárcel P. Influencia de las variables de carácter sociodemográfico sobre el síndrome de Burnout: un estudio en una muestra de profesionales de enfermería. Revista de Psicología Social Aplicada, 1996;6(2):43-63.

(16) Ríos Risquez MI, Peñalver Hernández F, Godoy Fernández C. Burnout y salud percibida en profesionales de enfermería de Cuidados Intensivos. Enferm Intensiva 2008; 19(4):169-78.

(17) García Grau N, Carmona Heredia A, Roca Biosca A, Olona Cabassés M. Síndrome de Burnout en los equipos de Enfermería de Cuidados Intensivos de Cataluña. Metas 2004; 7(2):6-12.

(18) Carmona Monge FJ, Sanz Rodríguez LJ, Marín Morales D. Relaciones entre el Síndrome de Burnout en profesionales de enfermería. Factores sociodemográficos y reactividad al estrés. Enfermería Científica 2002; ene-feb.238-239:33-39.

(19) Santana Cabrera L, Hernández Medina E, Eugenio Robaina P, Sánchez-Palacios M, Pérez Sánchez R, Falcón Moreno R. Síndrome de burnout entre el personal de enfermería y auxiliar de una unidad de cuidados intensivos y el de las plantas de hospitalización. Enferm Clin 2009; 19(1):31-34. 


\section{ANEXO I}

\begin{tabular}{|c|c|c|}
\hline CARACTERÍTICAS GENERALES & LA MUESTRA & \\
\hline CATEGORÍA & DUE & $75 \%(\mathrm{~N}=51)$ \\
\hline & $\mathrm{AE}$ & $25 \%(\mathrm{~N}=17)$ \\
\hline SEXO & MUJER & $86,7 \%(\mathrm{~N}=59)$ \\
\hline & HOMBRE & $13,3 \%(\mathrm{~N}=9)$ \\
\hline EDAD & $<25$ años & $14,7 \%(\mathrm{~N}=10)$ \\
\hline & 25-34 años & $20,6 \%(\mathrm{~N}=14)$ \\
\hline & 35-44 años & $32,3 \%(\mathrm{~N}=22)$ \\
\hline & 45-54 años & $28 \%(\mathrm{~N}=19)$ \\
\hline & $\geq 55$ años & $4,4 \%(N=3)$ \\
\hline TIPO DE CONTRATO & FIJO & $51,5 \%(\mathrm{~N}=35)$ \\
\hline & INTERINO & $8,8 \%(\mathrm{~N}=6)$ \\
\hline & EVENTUAL & $39,7 \%(\mathrm{~N}=27)$ \\
\hline EXPERIENCIA LABORAL & $<5$ años & $26,5 \%(\mathrm{~N}=18)$ \\
\hline & 5-10 años & $14,7 \%(\mathrm{~N}=10)$ \\
\hline & 11-15 años & $3 \%(\mathrm{~N}=2)$ \\
\hline & $16-20$ años & $27,9 \%(\mathrm{~N}=19)$ \\
\hline & $\geq 21$ años & $27,9 \%(\mathrm{~N}=19)$ \\
\hline ANTIGÜEDAD EN EL PUESTO & $<1$ año & $26,5 \%(\mathrm{~N}=18)$ \\
\hline & 1-5 años & $20,6 \%(\mathrm{~N}=14)$ \\
\hline & 6-10 años & $11,7 \%(\mathrm{~N}=8)$ \\
\hline & 11-15 años & $17,6 \%(\mathrm{~N}=12)$ \\
\hline & $16-20$ años & $1,5 \%(\mathrm{~N}=1)$ \\
\hline & $\geq 21$ años & $22,1 \%(\mathrm{~N}=15)$ \\
\hline TURNO & ROTATORIO & $100 \%(\mathrm{~N}=68)$ \\
\hline CE & ALTO & $19,1 \%(\mathrm{~N}=13)$ \\
\hline & MEDIO & $20,6 \%(\mathrm{~N}=14)$ \\
\hline & BAJO & $60,3 \%(\mathrm{~N}=41)$ \\
\hline DP & ALTO & $23,5 \%(\mathrm{~N}=16)$ \\
\hline & MEDIO & $30,9 \%(\mathrm{~N}=21)$ \\
\hline & BAJO & $45,6 \%(\mathrm{~N}=31)$ \\
\hline $\mathrm{RP}$ & ALTO & $29,4 \%(\mathrm{~N}=20)$ \\
\hline & MEDIO & $30,9 \%(\mathrm{~N}=21)$ \\
\hline & BAJO & $39,7 \%(\mathrm{~N}=27)$ \\
\hline FASE & SB & $14,7 \%(\mathrm{~N}=10)$ \\
\hline & TB & $45,6 \%(\mathrm{~N}=31)$ \\
\hline & $A B$ & $39,7 \%(\mathrm{~N}=27)$ \\
\hline
\end{tabular}

\section{ANEXO II}

\section{NIVELES DIMENSIONES}

$\begin{array}{ll}\text { CANSANCIO } & \text { DESPERSONALIZACIÓN } \\ \text { EMOCIONAL (CE) } & \text { (DP) }\end{array}$

ALTO 25-54 puntos

10-30 puntos

4-9 puntos

0-3 puntos
REALIZACIÓN PERSONAL (RP)

40-48 puntos

34-39 puntos

0-33 puntos 
ANEXO III

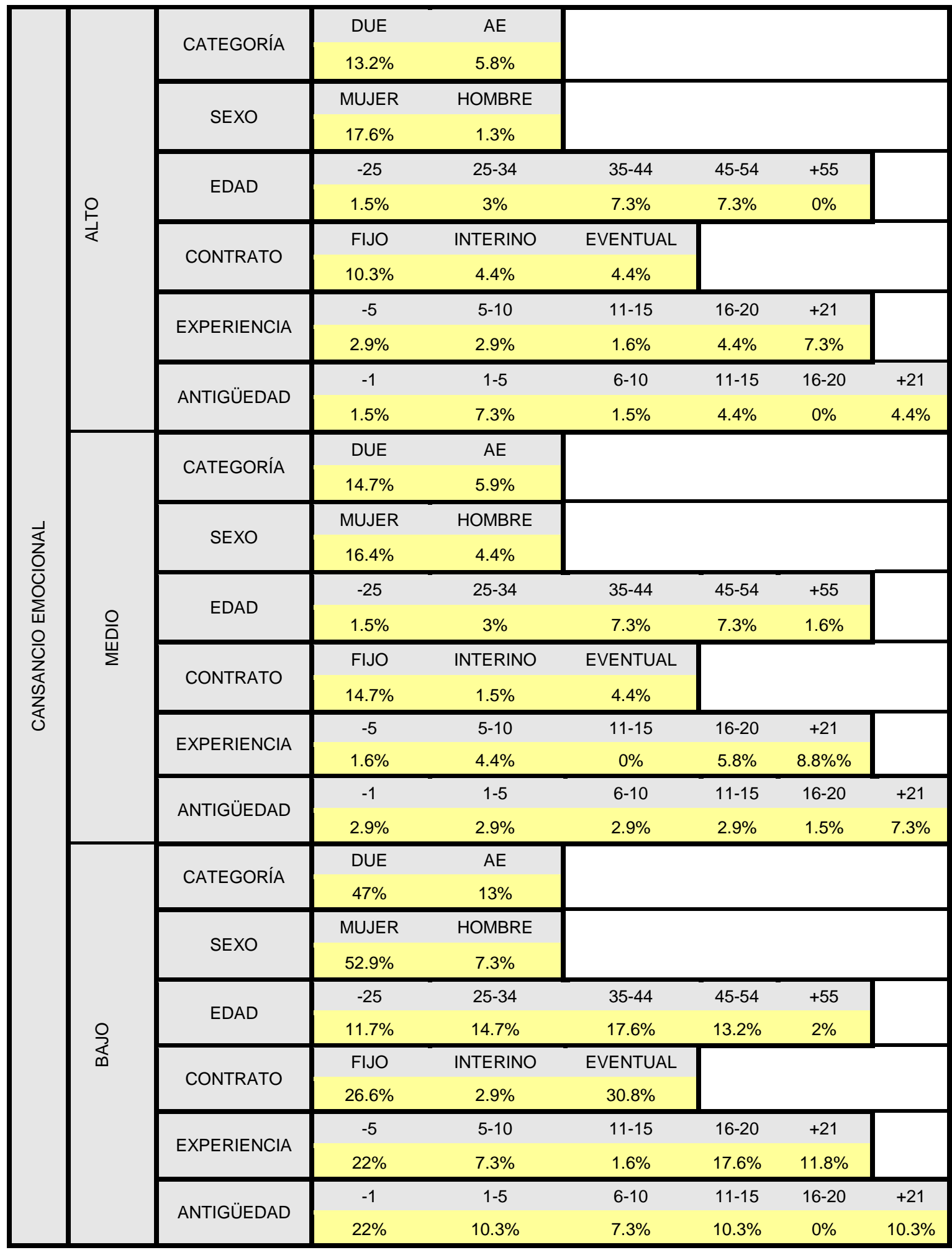


ANEXO IV

\begin{tabular}{|c|c|c|c|c|c|c|c|c|}
\hline & & CATEGORÍA & $\begin{array}{c}\text { DUE } \\
16.1 \%\end{array}$ & $\begin{array}{c}\mathrm{AE} \\
7.4 \%\end{array}$ & & & & \\
\hline & & cᄃY & MUJER & HOMBRE & & & & \\
\hline & & & $23.5 \%$ & $0 \%$ & & & & \\
\hline & & & -25 & $25-34$ & $35-44$ & $45-54$ & +55 & \\
\hline & 은 & LUTा & $1.5 \%$ & $7.3 \%$ & $8.8 \%$ & $6 \%$ & $0 \%$ & \\
\hline & $\vec{\varangle}$ & CONTRATO & FIJO & INTERINO & EVENTUAL & & & \\
\hline & & & $11.7 \%$ & $1.5 \%$ & $10.3 \%$ & & & \\
\hline & & EYPEPIENCIA & -5 & $5-10$ & $11-15$ & $16-20$ & +21 & \\
\hline & & & $5.8 \%$ & $5.8 \%$ & $1.6 \%$ & $4.5 \%$ & $5.8 \%$ & \\
\hline & & 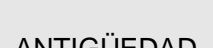 & -1 & $1-5$ & $6-10$ & $11-15$ & $16-20$ & +21 \\
\hline & & 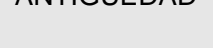 & $5.8 \%$ & $10.3 \%$ & $1.5 \%$ & $0 \%$ & $0 \%$ & $5.8 \%$ \\
\hline & & & DUE & $\mathrm{AE}$ & & & & \\
\hline & & 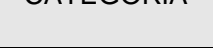 & $25 \%$ & $6 \%$ & & & & \\
\hline & & SEXO & MUJER & HOMBRE & & & & \\
\hline 0 & & & $23.5 \%$ & $7.3 \%$ & & & & \\
\hline 곤 & & & -25 & $25-34$ & $35-44$ & $45-54$ & +55 & \\
\hline$\sum_{0}^{5}$ & 음 & LERD & $4.4 \%$ & $4.4 \%$ & $10.2 \%$ & $10.2 \%$ & $1.5 \%$ & \\
\hline 㞻 & $\Sigma$ & CONTRATO & FIJO & INTERINO & EVENTUAL & & & \\
\hline 品 & & & $19.2 \%$ & $1.5 \%$ & $10.3 \%$ & & & \\
\hline & & EXPERIFNCIA & -5 & $5-10$ & $11-15$ & $16-20$ & +21 & \\
\hline & & 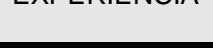 & $5.8 \%$ & $3 \%$ & $0 \%$ & $8.8 \%$ & $13.2 \%$ & \\
\hline & & ANTIGÜEDAD & -1 & $1-5$ & $6-10$ & $11-15$ & $16-20$ & +21 \\
\hline & & 1610 & $4.4 \%$ & $4.4 \%$ & $5.8 \%$ & $5.8 \%$ & $1.5 \%$ & $8.8 \%$ \\
\hline & & CATFGORÍA & DUE & $\mathrm{AE}$ & & & & \\
\hline & & 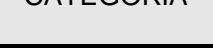 & $33.8 \%$ & $11.7 \%$ & & & & \\
\hline & & SEYO & MUJER & HOMBRE & & & & \\
\hline & & & $40 \%$ & $5.8 \%$ & & & & \\
\hline & & FDAR & -25 & $25-34$ & $35-44$ & $45-54$ & +55 & \\
\hline & $\stackrel{\circ}{?}$ & LLח & $8.8 \%$ & $8.8 \%$ & $11.7 \%$ & $11.7 \%$ & $3 \%$ & \\
\hline & 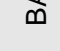 & CONTRATO & FIJO & INTERINO & EVENTUAL & & & \\
\hline & & (c) & $20.5 \%$ & $5.8 \%$ & $19.2 \%$ & & & \\
\hline & & EYDEDIENCIA & -5 & $5-10$ & $11-15$ & $16-20$ & +21 & \\
\hline & & & $14.7 \%$ & $5.8 \%$ & $1.6 \%$ & $14.8 \%$ & $8.8 \%$ & \\
\hline & & ANTIGÏIFDAD & -1 & $1-5$ & $6-10$ & $11-15$ & $16-20$ & +21 \\
\hline & & & $16.1 \%$ & $5.8 \%$ & $4.4 \%$ & $11.7 \%$ & $0 \%$ & $7.3 \%$ \\
\hline
\end{tabular}


ANEXO V

\begin{tabular}{|c|c|c|c|c|c|c|c|c|}
\hline & & CATFGORÍA & DUE & $A E$ & & & & \\
\hline & & & $19.1 \%$ & $10.3 \%$ & & & & \\
\hline & & cFY & MUJER & HOMBRE & & & & \\
\hline & & & $29.6 \%$ & $1.5 \%$ & & & & \\
\hline & & ו & -25 & $25-34$ & $35-44$ & $45-54$ & +55 & \\
\hline & 은 & & $4.4 \%$ & $6 \%$ & $14.7 \%$ & $4.4 \%$ & $0 \%$ & \\
\hline & $\overrightarrow{<}$ & CONTRATO & FIJO & INTERINO & EVENTUAL & & & \\
\hline & & & $14.7 \%$ & $2.9 \%$ & $11.8 \%$ & & & \\
\hline & & 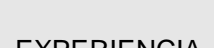 & -5 & $5-10$ & $11-15$ & $16-20$ & +21 & \\
\hline & & Lar Latioturt & $8.8 \%$ & $3 \%$ & $0 \%$ & $11.7 \%$ & $6.8 \%$ & \\
\hline & & חАТT: & -1 & $1-5$ & $6-10$ & $11-15$ & $16-20$ & +21 \\
\hline & & 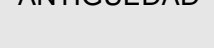 & $8.8 \%$ & $4.4 \%$ & $3 \%$ & $10.3 \%$ & $0 \%$ & $1.5 \%$ \\
\hline & & CATCOPDín & DUE & $\mathrm{AE}$ & & & & \\
\hline & & & $26.4 \%$ & $4.4 \%$ & & & & \\
\hline & & SFXO & MUJER & HOMBRE & & & & \\
\hline O & & 는 & $22 \%$ & $7.3 \%$ & & & & \\
\hline 㐍 & & חמתמת & -25 & $25-34$ & $35-44$ & $45-54$ & +55 & \\
\hline$z$ & 음 & LERD & $4.4 \%$ & $7.3 \%$ & $7.3 \%$ & $8.8 \%$ & $3 \%$ & \\
\hline$\frac{0}{\mathbb{S}}$ & $\dot{\Sigma}$ & CONTRATO & FIJO & INTERINO & EVENTUAL & & & \\
\hline $\bar{\rightleftarrows}$ & & & $16.1 \%$ & $1.5 \%$ & $13.3 \%$ & & & \\
\hline & & EXPERIENCIA & -5 & $5-10$ & $11-15$ & $16-20$ & +21 & \\
\hline & & & $7.3 \%$ & $4.5 \%$ & $0 \%$ & $7.3 \%$ & $11.7 \%$ & \\
\hline & & ANTTGÏ̈ЕRAR & -1 & $1-5$ & $6-10$ & $11-15$ & $16-20$ & +21 \\
\hline & & & $7.3 \%$ & $5.8 \%$ & $5.8 \%$ & $1.5 \%$ & $1.5 \%$ & $8.8 \%$ \\
\hline & & CATESORÍA & DUE & $\mathrm{AE}$ & & & & \\
\hline & & 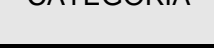 & $29.4 \%$ & $10.3 \%$ & & & & \\
\hline & & SEXO & MUJER & HOMBRE & & & & \\
\hline & & & $35.4 \%$ & $4.2 \%$ & & & & \\
\hline & & FDAR & -25 & $25-34$ & $35-44$ & $45-54$ & +55 & \\
\hline & 을 & LEח & $6 \%$ & $7.3 \%$ & $10.2 \%$ & $14.7 \%$ & $1.5 \%$ & \\
\hline & $\infty$ & CONTRATO & FIJO & INTERINO & EVENTUAL & & & \\
\hline & & ectritro & $20.5 \%$ & $\underline{4.4 \%}$ & $14.7 \%$ & & & \\
\hline & & EYPERIENCIA & -5 & $5-10$ & $11-15$ & $16-20$ & +21 & \\
\hline & & 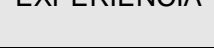 & $10.3 \%$ & $7.3 \%$ & $3 \%$ & $8.8 \%$ & $10.3 \%$ & \\
\hline & & АNTIGÏ̈RAR & -1 & $1-5$ & $6-10$ & $11-15$ & $16-20$ & +21 \\
\hline & & & $10.3 \%$ & $10.3 \%$ & $3 \%$ & $5.8 \%$ & $0 \%$ & $10.3 \%$ \\
\hline
\end{tabular}


ANEXO VI

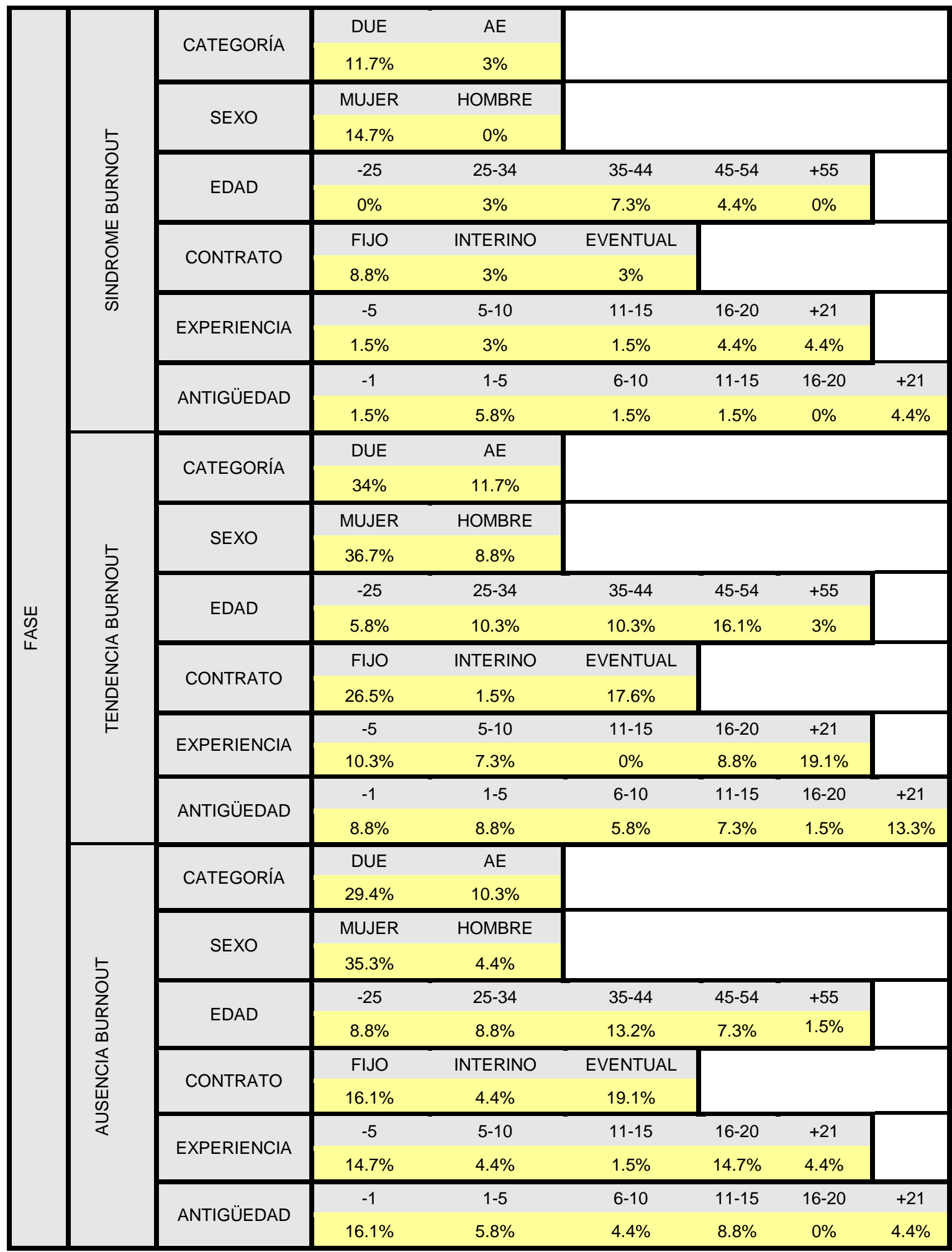

ISSN 1695-6141 